\title{
Coalescence-driven magnetic order of the uncompensated antiferromagnetic Co doped $\mathrm{ZnO}$
}

\author{
V. Ney, ${ }_{1}^{1, *}$ B. Henne, ${ }^{1}$ J. Lumetzberger, ${ }^{1}$ F. Wilhelm,,${ }^{2}$ K. Ollefs, ${ }^{2}$ A. Rogalev, ${ }^{2}$ A. Kovacs, ${ }^{3}$ M. Kieschnick, ${ }^{4}$ and A. Ney ${ }^{1}$ \\ ${ }^{1}$ Institut für Halbleiter- und Festkörperphysik, Johannes Kepler Universität, Altenbergerstraße 69, 4040 Linz, Austria \\ ${ }^{2}$ ESRF - The European Synchrotron, CS 40220, 38043 Grenoble Cedex 9, France \\ ${ }^{3}$ Ernst Ruska-Centre for Microscopy and Spectroscopy with Electrons, Peter Grünberg Institut 5 , \\ Forschungszentrum Jülich, 52425 Jülich, Germany \\ ${ }^{4}$ Ruhr-Universität Bochum, RUBION, Universitätsstraße 150, D-44801 Bochum, Germany
}

(Received 14 September 2016; published 5 December 2016)

\begin{abstract}
The evolution of the structural and magnetic properties of $\mathrm{Co}$ doped $\mathrm{ZnO}$ has been investigated over an unprecedented concentration range above the coalescence limit. $\mathrm{ZnO}$ films with Co concentrations from $20 \%$ to $60 \%$ of the cationic lattice have been grown by reactive magnetron sputtering. The wurtzite crystal structure was maintained even for these high dopant concentrations. By measuring the x-ray absorption at the near edge and the linear and circular dichroism of the films at the $\mathrm{Zn}$ and Co $K$ edge, it could be shown that Co substitutes predominantly for $\mathrm{Zn}$ in the lattice. No indications of metallic Co have been found in the samples. At low Co concentrations, the films are paramagnetic, but with increasing Co content, the films become antiferromagnetically ordered with increasing order temperature. Uncompensated spins, coupled to the antiferromagnetic dopant configurations, lead to a vertical exchange-bias-like effect, which increases with increasing Co concentration. In parallel, the single-ion anisotropy is gradually lost.
\end{abstract}

DOI: 10.1103/PhysRevB.94.224405

\section{INTRODUCTION}

There have been several approaches to tailor the magnetic properties of $\mathrm{ZnO}$ to obtain a dilute magnetic semiconductor (DMS) after theory suggested its suitability [1]. Doping with elements such as $\mathrm{Co}$, Gd, and $\mathrm{Mn}$ seemed to be promising [2-5], as well as carrier-induced ferromagnetism [6]. However, reproducibility of the resulting magnetic properties was problematic from the beginning, as already pointed out in the conclusions of the first report on room-temperature ferromagnetism in $\mathrm{Zn}_{1-x} \mathrm{Co}_{x} \mathrm{O}$ (Co: $\left.\mathrm{ZnO}\right)$ [2]. Meanwhile, there is growing consensus that in cases where the Co dopant atoms substitute for $\mathrm{Zn}$ in the wurtzite lattice, the system exhibits paramagnetism with moments corresponding to the dopant atoms [7-9]. Moreover, due to the anisotropic wurtzite host crystal, the paramagnetism is also anisotropic $[7,10]$ and can be described by a well-established effective spin Hamiltonian introduced for the evaluation of ferromagnetic resonance [11] and optical investigations [12]. The uniaxial single-ion anisotropy $D S_{z}^{2}$ in the impurity limit was experimentally found to be $D / k_{B}=4 \mathrm{~K}[11,12]$. This is in good agreement with Co doping of a few percent [7] and with modern crystal-field theory [13]. This value was determined to be reduced for Co concentrations of $10 \%$ to $D / k_{B}=3 \mathrm{~K}$, and $2 \mathrm{~K}$ for $15 \%$ [10]. In addition, an antiferromagnetic coupling between next-cation-neighbor Co dopant atoms has already been reported for bulk $\mathrm{Co}: \mathrm{ZnO}$ crystals from early on [14-17], as well as later for epitaxial films $[9,18]$. The strength of the antiferromagnetic coupling could be quantified

\footnotetext{
*verena.ney@jku.at

Published by the American Physical Society under the terms of the Creative Commons Attribution 3.0 License. Further distribution of this work must maintain attribution to the author(s) and the published article's title, journal citation, and DOI.
}

using high-field magnetometry to be $J / k_{B}=15 \mathrm{~K}$ [19], which is in good agreement with ab initio calculations [20]. The phase-pure $\mathrm{Co}: \mathrm{ZnO}$ system is thus an anisotropic paramagnet with antiferromagnetic next-cation-neighbor exchange interactions. Altering the growth conditions for $\mathrm{Co}: \mathrm{ZnO}$ has led to films which showed clustering of the dopant atoms, resulting in a superparamagnetic behavior due to metallic Co precipitations $[21,22]$. The latter could be directly correlated with the magnetic properties of the $\mathrm{Co}: \mathrm{ZnO}$ films [23]. In other works, the presence of ferromagnetic order in $\mathrm{Co:ZnO}$ was correlated with the presence of defects [24], which, however, could not be corroborated by systematic variations of the preparation conditions or defects induced by ion implantation [25].

To explain the appearance of ferromagnetic order in $\mathrm{Co}: \mathrm{ZnO}$, another path was suggested by theory, namely, coalescence-driven magnetic order [26]. For dopants in the hcp sublattice of the wurtzite structure of $\mathrm{ZnO}$, coalescence occurs at about 20\% [27]. Consistent with this, it was calculated that starting from $20 \%$ up to $60 \% \mathrm{Co}: \mathrm{ZnO}$ ferromagnetic ordering will be observed with an order temperature increasing to around $240 \mathrm{~K}$ [26]. Following a transition region around $70 \%$, between 80 and $100 \%$ Co content layerwise antiferromagnetism was predicted [26]. In the extreme limit of $100 \% \mathrm{Co:ZnO}$ (wurtzite $\mathrm{CoO}$ ) [26], a Néel temperature of $T_{N}=300 \mathrm{~K}$ is reached [see Fig. 8(d)]. Generally, at concentrations above $50 \%$, the films can be considered to be wurtzite $\mathrm{CoO}$ with $\mathrm{Zn}$ doping. However, $\mathrm{CoO}$ itself usually stabilizes in a cubic crystal structure. The first report on its hexagonal occurrence has been in a mixed powder [28]. The growth of wurtzite $\mathrm{CoO}$ films on $\mathrm{ZnO}$ buffer layers has also been reported [29]. They were found to be paramagnetic, which was explained by the geometric frustration of the antiferromagnetic superexchange, and were observed to be structurally/chemically unstable [29]. Nanocrystals/areas of $5 \%$ wurtzite $\mathrm{CoO}$ embedded in a $\mathrm{ZnO}$ host crystal have also been investigated [30]. Uncompensated spins at the surface of these antiferromagnetic nanocrystals/areas were suggested 
to be responsible for ferromagnetic signatures in these films, with a reduced effective magnetic moment per Co, depending on the shape and size of the nanocrystal [30]. There are only a few reports where higher percentages of $\mathrm{Co}$ in a wurtzite $\mathrm{ZnO}$ host crystal have been stabilized. The good dopability of $\mathrm{ZnO}$ with $\mathrm{Co}$ has been shown for films with Co concentrations at the coalescence limit 20\% [31]. Based on synchrotron methods, quality indicators for $\mathrm{Co}: \mathrm{ZnO}$ were introduced [22], which allow one to prove the substitutional incorporation of the $\mathrm{Co}$ in the lattice and thus the absence of phase separation. However, these films were still paramagnetic [31]. Higher Co concentrations of up to $40 \%$ were realized in a polycrystalline film via a digital alloy, i.e., a $\mathrm{ZnO} / \mathrm{CoO}$ multilayer [32].

Recenty, we reported on the successful synthesis of $60 \%$ $\mathrm{Co}: \mathrm{ZnO}$, which is one of the highest concentrations for which the wurtzite structure of $\mathrm{ZnO}$ is still retained [33]. For these $\mathrm{Co}: \mathrm{ZnO}$ films, a vertical exchange bias has been observed [34], which was explained by uncompensated spins of otherwise antiferromagnetic regions with a broad size distribution of the Co dopant configurations [34]. At these high dopant concentrations, the distribution of Co atoms in the lattice becomes the dominating factor in the evolution of the resulting magnetic properties from coalescence up to $60 \% \mathrm{Co}$.

Assuming a statistical dopant distribution in the hcp cationic sublattice of the wurtzite structure, for rather low concentrations, Behringer's equations [35] can be used (see, e.g., the discussions in Refs. $[9,10,19])$. They allow one to calculate the abundances of isolated Co dopant atoms (singles), Co-O-Co pairs, and open and closed Co-O-Co-O-Co triples. However, with increasing doping level, larger configurations cannot be neglected. These are not accessible by theory beyond either coherent phase approximation (CPA), which disregards disorder, or supercell calculations, where the accessible size is rather limited. The high dopability of $\mathrm{ZnO}$ with $\mathrm{Co}$ provides an experimental model system, which can be used to study the magnetic properties of such films with increasing Co concentration, where extremely large dopant configurations occur.

Here we report on the growth, structure, and magnetic properties of $\mathrm{Co}: \mathrm{ZnO}$ films covering the concentration range from coalescence at $20 \%$ to $60 \%$ to compare the experimental findings with the theoretically predicted phase diagram of Ref. [26]. The evolution of structure and a comprehensive set of magnetic properties has been analyzed along the concentration series by integral and element-selective synchrotron methods based on x-ray absorption spectroscopy.

\section{EXPERIMENT}

$\mathrm{Co}: \mathrm{ZnO}$ films with nominal Co concentrations from $20 \%$ up to $60 \%$ of $\mathrm{Co}$ in $\mathrm{ZnO}$ have been grown on $c$-plane sapphire (0001) by dc reactive magnetron sputtering at a power of $20 \mathrm{~W}$. Metallic targets with 20,30, and 50\% of Co in $\mathrm{Zn}$ have been used in combination with a sputter gas composition of $\mathrm{Ar}: \mathrm{O}_{2}$ of 10:1 standard cubic centimeters per minute $(\mathrm{sccm})$ regulated by separate mass flow controllers. This composition of the sputter gas as well as the substrate temperature of $350{ }^{\circ} \mathrm{C}$ have been optimized to obtain the best possible structural properties. A ceramic composite target of $\mathrm{ZnO}$ and $\mathrm{Co}_{3} \mathrm{O}_{4}$ (ratio 3:2) has been sputtered at $30 \mathrm{~W}$ with only Ar, and the substrate temperature was kept at $525^{\circ} \mathrm{C}$ which results in the $60 \% \mathrm{Co}: \mathrm{ZnO}$ as reported before [33]. The actual concentration of Co in the films was determined with particle-induced $\mathrm{X}$-ray emission (PIXE) and energy dispersive $\mathrm{X}$-ray fluorescence (EDX). This yielded concentrations of $19.6 \%$ and $35.0 \%$ using PIXE and $60 \%$ using EDX, respectively. The thicknesses of the samples was measured with $\mathrm{X}$-ray reflectometry (XRR) to be between $152 \pm 3 \mathrm{~nm}(20 \%)$ and $216 \pm 4 \mathrm{~nm}(60 \%)$. For $20 \%$ of Co also a $1-\mu \mathrm{m}$-thick film has been investigated for comparison, but the thickness had no influence on the structural or magnetic properties.

To analyze the global structural properties, XRR and $\mathrm{X}$-ray diffraction (XRD) measurements were done with a PANalytical X'pert MRD XL diffractometer to record $\omega-2 \theta$ as well as $\omega$-rocking scans. In addition, a transmission electron microscopy (TEM) specimen has been prepared from the $35 \%$ sample according to the procedure described in more detail in Ref. [36].

A commercial superconducting quantum interference device (SQUID) magnetometer (Quantum Design MPMS-XL) was used for the integral magnetic investigations. If not otherwise mentioned, the samples were studied with the magnetic field applied in the film plane (IP) and all measurement artifacts as described in Refs. [37-39] were corrected; signals below $2 \cdot 10^{-7}$ emu were disregarded. The diamagnetic background from the sapphire substrate has been subtracted from all measurements. $\mathrm{M}(\mathrm{H})$ curves were taken at 300 and $2 \mathrm{~K}$, as well as field-cooled (FC), zero-field-cooled (ZFC), and field-heated (FH) measurements. For the FH measurements, the sample was cooled in a large field of $5 \mathrm{~T}$ and the $\mathrm{M}(\mathrm{T})$ curves have been measured at $10 \mathrm{mT}$, while warming the sample from 2 to $300 \mathrm{~K}$. For the ZFC data, the sample is cooled without an applied field and the $\mathrm{M}(\mathrm{T})$ curve is measured while warming up from 2 to $300 \mathrm{~K}$ under a magnetic field of $10 \mathrm{mT}$. The FC data was recorded subsequently while cooling the sample again to $2 \mathrm{~K}$ in the same measurement field of $10 \mathrm{mT}$. To determine the anisotropy, IP and out-of-plane (OOP) measurements have been conducted on the same sample piece which was rotated in the same straw to minimize artifacts $[38,39]$.

Element-specific measurements were done by using x-ray absorption near-edge spectra (XANES), X-ray linear dichroism (XLD), and X-ray magnetic circular dichroism (XMCD). For the $\mathrm{Zn}$ and Co $K$ edges, XANES were taken at the European Synchrotron Radiation Facility (ESRF), beam line ID12, in total fluorescence yield in backscattering geometry and all absorption spectra were normalized with respect to the edge jump. For the XLD measurements, a quarter-wave plate was used to flip the linear polarization of the synchrotron light from vertical to horizontal, as described in greater detail elsewhere [40,41]; the angle of incidence was $10^{\circ}$ with respect to the sample surface. The XMCD measurements were taken as the direct difference of XANES spectra recorded with right and left circular polarized light under grazing incidence $\left(15^{\circ}\right)$. The XMCD spectra were recorded in an external magnetic field of up to $17 \mathrm{~T}$ provided by a superconducting magnet. To minimize artifacts, the external field was reversed as well. $\mathrm{XMCD}(\mathrm{H})$ curves were taken at fixed photon energies as a function of the external magnetic field, while the helicity of the light was reversed at each field point. 

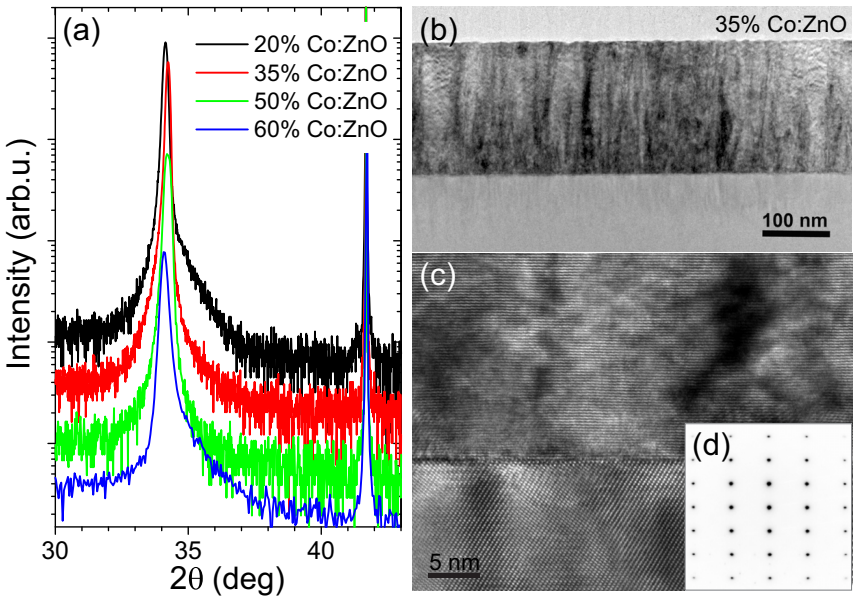

FIG. 1. Global structural analysis shows the wurtzite crystal structure of all films. (a) XRD $2 \theta-\omega$ scans. (b), (c) TEM image of the $35 \% \mathrm{Co}: \mathrm{ZnO}$ sample. (d) The corresponding electron-diffraction pattern.

\section{STRUCTURAL PROPERTIES}

Figure 1(a) shows the $2 \theta-\omega$ scans for the samples in the range of $30^{\circ}$ to $45^{\circ}$. In this range, only two peaks are observed: the (006) substrate peak of $\mathrm{Al}_{2} \mathrm{O}_{3}$ and the (002) diffraction resulting from the $\mathrm{Co}: \mathrm{ZnO}$ films. For undoped $\mathrm{ZnO}(001)$, the (002) diffraction is at $34.42^{\circ}$ corresponding to a lattice constant $c$ of $5.2071 \AA$ (Refs. [42,43]). The Co doped ZnO films have a slightly lower diffraction angle from $34.13^{\circ}$ to $34.25^{\circ}$, which corresponds to a lattice constant from 5.251 to $5.233 \AA$. The FWHM of the (002) diffraction ranges from $(0.14 \pm 0.01)^{\circ}$ to $(0.60 \pm 0.05)^{\circ}$, and from $0.72^{\circ}$ to $0.90^{\circ} \pm 0.05^{\circ}$ in the rocking $(\omega)$ scans (not shown). No other diffraction peaks occur in the range of $15^{\circ}$ to $70^{\circ}$ for any of the samples. To summarize the XRD results, Co:ZnO grows highly textured, with the $c$ axis of the film being perpendicular to the sample surface with a slightly reduced crystalline quality for increasing $\mathrm{Co}$ concentration.

The TEM image taken on the 35\% Co: $\mathrm{ZnO}$ sample [Fig. 1(b)] shows the typical columnar growth of the $\mathrm{Co}: \mathrm{ZnO}$ films [36]. The layer thickness of around $200 \mathrm{~nm}$ can be confirmed as well as the smooth surface. Even though the grains are slightly misoriented with respect to each other, the layer is continuous [Fig. 1(c)], but the strain contrast is strong and shows that there are some stacking faults and dislocations. The interface between the $\mathrm{Al}_{2} \mathrm{O}_{3}$ and the $35 \% \mathrm{Co}: \mathrm{ZnO}$ films is abrupt but moderately enriched with defects, but no indication of secondary phases can be seen. Compared to $10 \% \mathrm{Co:ZnO}$, the $20 \%$ films have an increased number of point defects [36], which is even further enhanced for higher concentrations and the misorientation of the grains is larger, which is consistent with the moderate increase of the FWHM of the XRD results for increasing Co content. The electron-diffraction pattern in Fig. 1(d) confirms a well-defined wurtzite crystal structure.

To obtain element-selective information of the local structural properties, XANES and XLD measurements at the Zn and Co $K$ edges have been recorded. In Fig. 2(a), the isotropic XANES (left scale) and the XLD (right scale) of the Co:ZnO samples are shown for the $\mathrm{Zn} K$ edge. In the isotropic

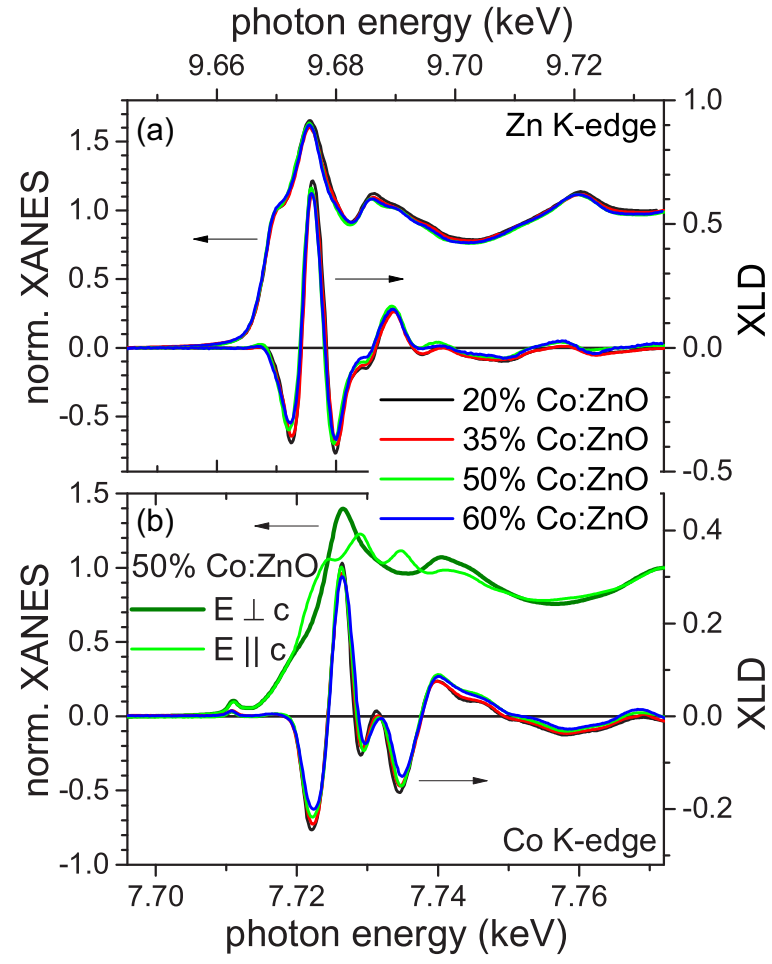

FIG. 2. (a) For all samples, the normalized isotropic XANES (left scale) at the $\mathrm{Zn} K$ edge and the corresponding XLD for the Co:ZnO samples (right scale) are shown. (b) The normalized XANES spectra for linear polarized light at the Co $K$ edge, once perpendicular to the $c$ axis of the sample and once parallel, is shown exemplarily for $50 \%$ $\mathrm{Co}: \mathrm{ZnO}$. The direct difference of these-the XLD—is plotted again for all samples.

XANES, hardly any differences between the samples can be observed. The clear difference in the XANES for linear polarized light parallel and perpendicular to the $c$ axis of the film is exemplarily displayed in Fig. 2(b) for the Co $K$ edge of the $50 \%$ sample, which is representative for the entire Co concentration range. The isotropic XANES at the Co $K$ edge for all samples can be found in Fig. 3(a). A crucial role for the analysis of the absorption edge is played by the size of the pre-edge feature of the XANES, which is only pronounced for $\mathrm{Co}$ in its $2+$ state, while its size is reduced for $\mathrm{Co}^{3+}$ and turns into a shoulder for Co metal $[23,33]$. For all samples discussed here, its size complies with the quality indicator "pre-edge" of 1.75 (see Fig. 2(c) of Ref. [22]), thus demonstrating that the samples are devoid of metallic Co. In addition, as for the $\mathrm{Zn}$ $K$ edge, no shift in photon energy and thus no change of the valence is observed at the Co $K$ edge.

Figure 2(b) also displays all XLD spectra for all samples and its signature is typical for the wurtzite crystal structure [44], i.e., tetrahedral coordination of $\mathrm{Zn}$ and $\mathrm{Co}$ to $\mathrm{O}$. The size of the XLD at both edges decreases only slightly with increasing Co concentration. This is a sign of a moderately reduced local crystalline structure, which is, however, consistent with the moderate reduction of the global crystalline perfection as evidenced by XRD. For the $20 \%$ sample, the XLD has a total amplitude of 0.58 , which is slightly smaller than the quality indicator of 0.60 , which was introduced for a concentration of $10 \%$ [22]. With increasing Co percentage, the size of the 
XLD at the Co $K$ edge is further reduced. However, one has to consider that the relation between the $\mathrm{Zn}$ and the Co $\mathrm{XLD}$ is also an important indicator. For all concentrations, this relation $\mathrm{XLD}(\mathrm{Co}) / \mathrm{XLD}(\mathrm{Zn})$ is $0.54 \pm 0.01$, which implies that the reduction of the Co XLD is as pronounced as for $\mathrm{Zn}$, which is in turn caused by a slight reduction of the global crystallinity as evidenced by XRD. The fact that the reduction of the XLD is observed for both elements to the same degree is thus no indication of the formation of secondary phases, but merely a consequence of a moderate degradation of the overall structural quality of the wurtzite crystal at these extreme Co doping levels.

\section{MAGNETIC PROPERTIES}

In Fig. 3, the normalized and isotropic XANES at the Co $K$ edge [Fig. 3(a)] and the corresponding XMCD measurements for the $\mathrm{Co}: \mathrm{ZnO}$ samples [Fig. 3(b)] are shown. The samples have been measured at $2.5 \mathrm{~K}$ and $17 \mathrm{~T}$. Two regions of the $\mathrm{XMCD}$ spectra are of special interest (marked with arrows), since they correspond to $\mathrm{Co}^{2+}(7.710 \mathrm{keV})$ and $\mathrm{Co}^{0}(\mathrm{Co}$ metal, $7.715 \mathrm{keV}$ ), respectively [22]. A clear reduction of the XMCD with increasing Co concentration can be observed at the pre-edge, i.e., for $\mathrm{Co}^{2+}$, whereas there is no significant XMCD signal at photon energies characteristic for Co metal. Consistent with the findings for the size of the pre-edge feature,

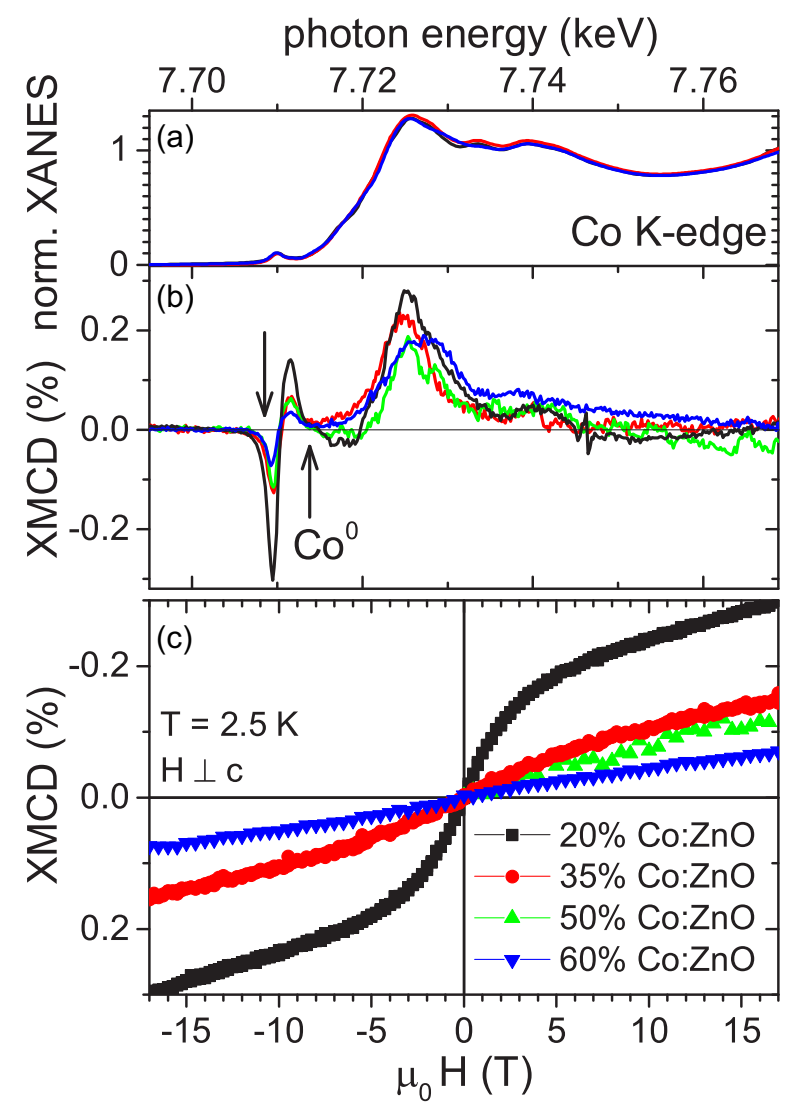

FIG. 3. (a) The normalized and isotropic XANES at the Co $K$ edge and (b) the corresponding XMCD are shown for all $\mathrm{Co}: \mathrm{ZnO}$ samples. (c) At $7.71 \mathrm{keV}$, the XMCD has been measured in dependence of an externally applied magnetic field at $2.5 \mathrm{~K}$.

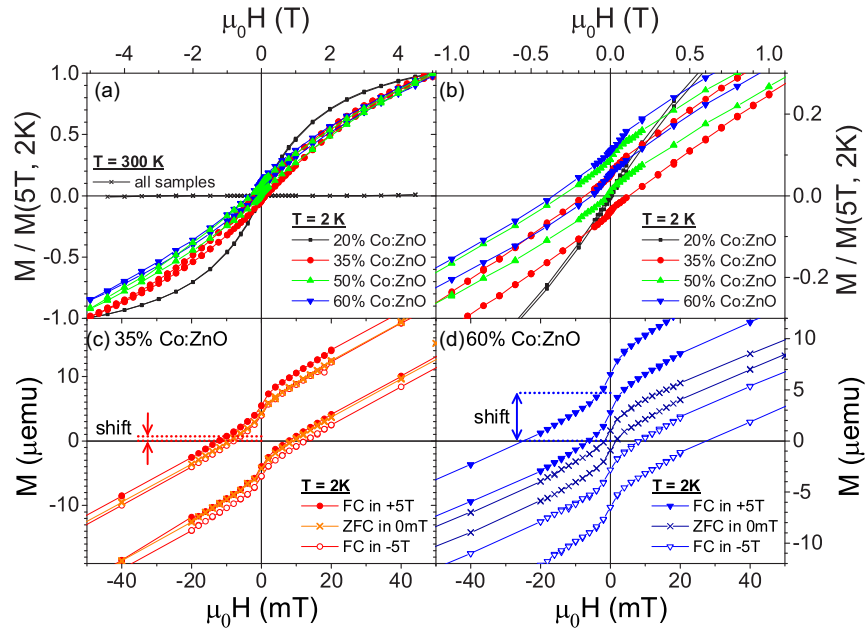

FIG. 4. (a) SQUID M(H) measurements at 2 and $300 \mathrm{~K}$, normalized to the magnetization measured at $5 \mathrm{~T}$ and $2 \mathrm{~K}$. (b) Enlargement of the low-field region. (c), (d) The vertical exchange-bias shift for the (c) $35 \%$ and (d) $60 \% \mathrm{Co:ZnO}$ samples. For these, the $\mathrm{M}(\mathrm{H})$ curves have been measured after being cooled in $+5 \mathrm{~T},-5 \mathrm{~T}$ and without applied field.

this confirms that all specimens are devoid of metallic Co and $\mathrm{Co}^{2+}$ is the only Co species in all samples. $\mathrm{XMCD}(\mathrm{H})$ curves for $\mathrm{Co}^{2+}$ at $7.710 \mathrm{keV}$ up to $17 \mathrm{~T}$ have been recorded which exhibit a nonsaturating behavior for all samples [Fig. 3(c)]. For the $20 \% \mathrm{Co}: \mathrm{ZnO}$ sample, a clear curvature for the $\mathrm{XMCD}(\mathrm{H})$ curve is visible, which is well known from paramagnetic $\mathrm{Co}: \mathrm{ZnO}$ with lower Co concentrations [19]. In contrast, at higher Co concentrations, the XMCD increases virtually linear with the applied field. Since the size of the XMCD is directly proportional to the effective magnetic moment per Co, a decreasing effective magnetic moment per Co can be deduced with increasing Co percentage. This will be discussed in more detail in the following.

The results of the SQUID $\mathrm{M}(\mathrm{H})$ measurements are summarized in Fig. 4(a). For easier comparison, the measurements are normalized to the magnetization at $5 \mathrm{~T}$ and $2 \mathrm{~K}$. For all samples, the signal at $300 \mathrm{~K}$ falls below the detection limit of the SQUID $\left(2 \times 10^{-7}\right.$ emu $)$ [37]. The $20 \%$ Co: $\mathrm{ZnO}$ sample shows an anhysteretic, $\mathrm{S}$-shaped $\mathrm{M}(\mathrm{H})$ curve, which is typical for paramagnetic samples [22]. The curvature for the other samples reduces with increasing Co content, consistent with the findings of a reduced effective magnetic moment. A closer look at the low-field region in Fig. 4(b) reveals that all samples except the $20 \% \mathrm{Co}: \mathrm{ZnO}$ exhibit an open hysteresis, which, in addition, is vertically shifted. This vertical exchange-bias effect has already been reported for $60 \% \mathrm{Co}: \mathrm{ZnO}$ and was attributed to uncompensated antiferromagnetism [34]. The effect has been explored for the entire concentration series and the results are exemplarily shown for the $35 \%$ and $60 \%$ samples. The $\mathrm{M}(\mathrm{H})$ curves have been measured after cooling the sample in an applied external field of $+5 \mathrm{~T},-5 \mathrm{~T}(\mathrm{FC})$ and $0 \mathrm{mT}$ (ZFC) which is shown in Figs. 4(c) and 4(d), respectively. Consistent with Ref. [34], the vertical shift is absent if the sample is cooled in $0 \mathrm{mT}$. Cooling in opposite applied fields shifts the $\mathrm{M}(\mathrm{H})$ curve in opposite directions without changing the shape. The presence of a training effect has already been 


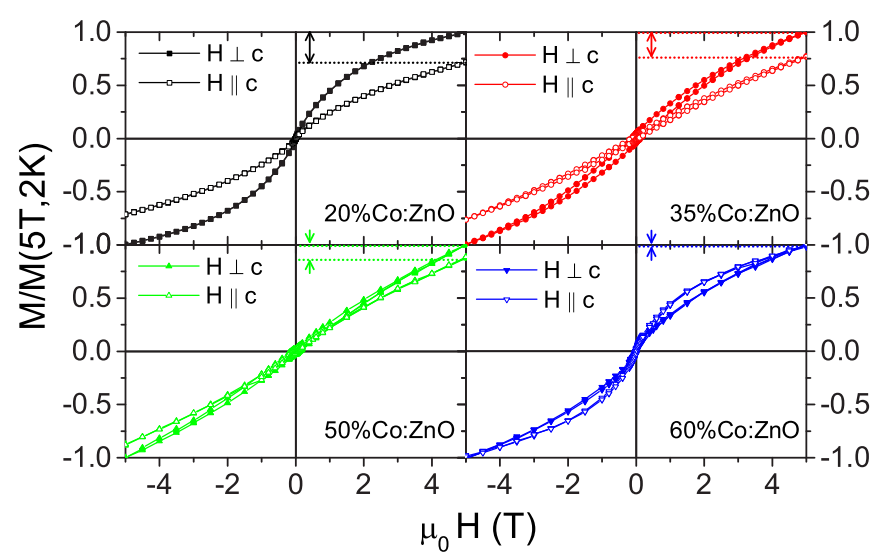

FIG. 5. IP vs OOP SQUID measurements at $2 \mathrm{~K}$. The arrows mark the size of the anisotropy at $5 \mathrm{~T}$.

reported for $60 \% \mathrm{Co:ZnO}$ in Ref. [34], Fig. 4. In addition to the vertical shift, a field-imprinted frustrated magnetization of about 3\% has been observed in the first $\mathrm{M}(\mathrm{H})$ cycle after FC, which vanished in the second cycle, thus leading to the same opening of the $\mathrm{M}(\mathrm{H})$ curves after $\mathrm{FC}$ and $\mathrm{ZFC}$.

In the next step, another characteristic feature of dilute Co in $\mathrm{ZnO}$, namely, the occurrence of anisotropic paramagnetism due to the uniaxial symmetry of the wurtzite structure of $\mathrm{ZnO}$ $[7,10]$, has been investigated. To determine the remaining effective single-ion anisotropy of $\mathrm{Co}: \mathrm{ZnO}$ films above the coalescence limit, $\mathrm{M}(\mathrm{H})$ curves have been recorded with the SQUID applying the magnetic field parallel (OOP) and perpendicular (IP) to the $c$ axis of $\mathrm{ZnO}$. For easier comparison, the data in Fig. 5 are normalized to the IP magnetization at $5 \mathrm{~T}$ and $2 \mathrm{~K}$ and the size of the anisotropy is determined by the difference between $\mathrm{M}$ at $2 \mathrm{~K}$ and $5 \mathrm{~T}$ for IP and OOP (marked by arrows in Fig. 5). While at the coalescence limit of 20\% a clear anisotropy can be observed, it strongly reduces until it vanishes completely at $60 \%$. At this high concentration, only a slight difference in the curvature of the $\mathrm{M}(\mathrm{H})$ can be seen. It should be noted that the curvature of the $M(H)$ curve for OOP increases just slightly, while it strongly decreases for IP.

Finally, the robustness of the observed magnetic order shall be investigated as a function of Co concentration by recording $\mathrm{M}(\mathrm{T})$ curves under FH, FC, and ZFC conditions. The bifurcation between FC and ZFC curves allows one to determine the presence of a magnetic hysteresis, whereas the bifurcation between $\mathrm{FH}$ and $\mathrm{ZFC} / \mathrm{FC}$ also includes magnetic order due to uncompensated and/or frustrated antiferromagnetism. This is because the large cooling field for the FH curve allows one to maximize the size of the uncompensated moments. The arrows in Fig. 6 indicate the temperature where the FH and ZFC curves bifurcate, i.e., where the transition from an uncompensated antiferromagnet to a paramagnet takes place. For the 20\% sample, hardly any separation is observable, consistent with the absence of a hysteresis in the $\mathrm{M}(\mathrm{H})$ curves, whereas with increasing Co percentage this separation becomes clearer and is shifted up to higher temperatures. For $60 \% \mathrm{Co}: \mathrm{ZnO}$ up to $20 \mathrm{~K}$, the separation is obvious, which corresponds well with the next-cation-neighbor exchange strength of $15 \mathrm{~K}$ from Ref. [19], which underlines that longer-range magnetic

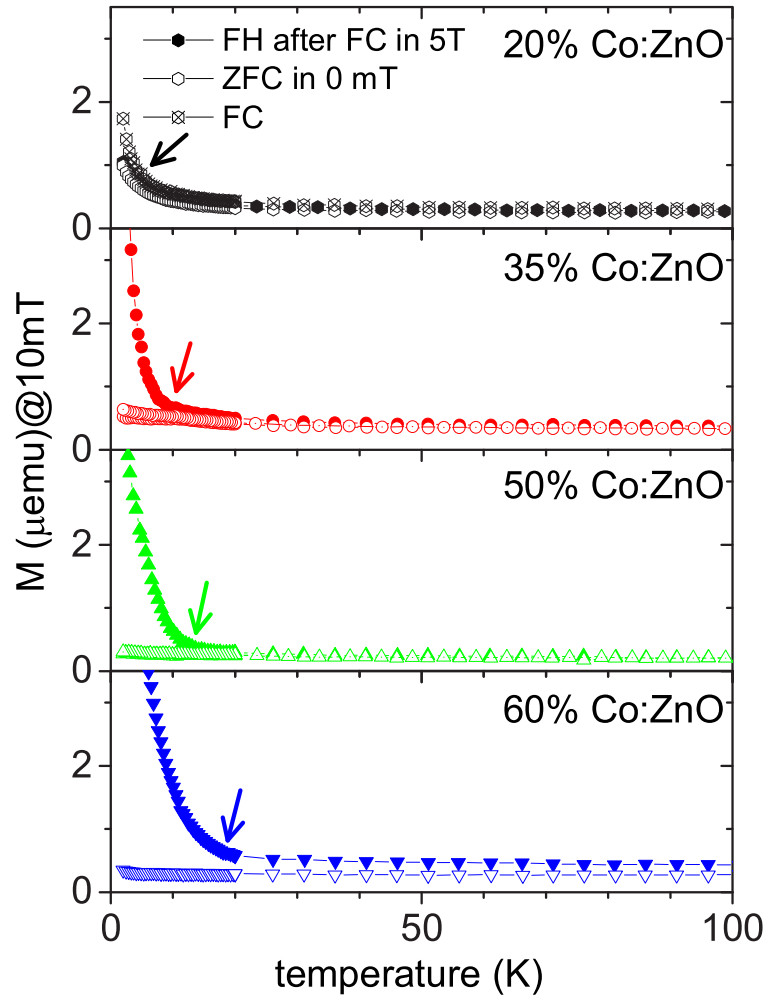

FIG. 6. SQUID M(T) measurements following the FC, FH, and ZFC procedures. A separation of the curves suggests magnetic order. The arrows indicate the temperature where the transition takes place.

interactions play no significant role in Co:ZnO. However, an additional weak separation between FH and ZFC for the $60 \%$ $\mathrm{Co}: \mathrm{ZnO}$ lasts up to around $200 \mathrm{~K}$, which is not present for $50 \%$ $\mathrm{Co}$ and below. This may be an indication that at these extreme doping levels, robust long-range magnetic order may set in, which should be investigated at even higher Co concentrations; unfortunately, so far this moderately $\mathrm{Zn}$ doped $\mathrm{CoO}$ in the wurtzite structure has not been successfully synthesized. Note that there are no samples in the series for which a significant bifurcation between the FC and ZFC curve exists which excludes a second, ferromagnetic component in all samples, which is again consistent with the absence of any metallic Co compound. On the other hand, this also excludes the presence of a coalescence-induced magnetic order at elevated temperatures.

\section{DISCUSSION}

The structural analysis of the $\mathrm{Co}: \mathrm{ZnO}$ samples shows that when increasing the Co percentage up to $60 \%$, the wurtzite crystal structure is maintained. The XRD and XLD show that this is accompanied by a slight reduction of the structural quality. Nevertheless, the reduction in size of the XLD at the $\mathrm{Zn} K$ edge and the Co $K$ edge are of the same order, which hints towards a general reduced crystal perfection and not to the formation of a secondary phase. In addition, in XANES and XMCD at the Co $K$ edge, only the typical spectral features of $\mathrm{Co}^{2+}$ in tetrahedral coordination are seen. Both at the pre-edge feature of the XANES as well as in the XMCD, the signature of metallic Co is missing. With all 


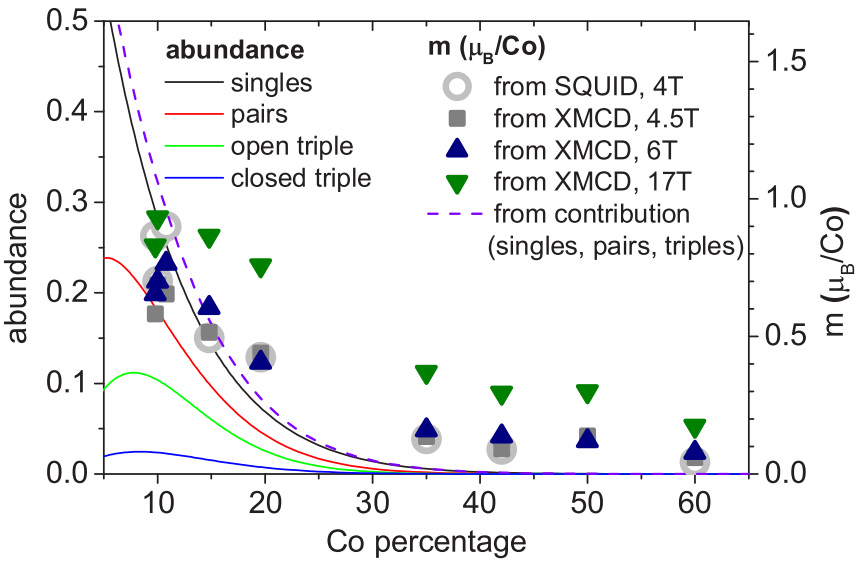

FIG. 7. The abundance of singles, pairs, and triples are displayed (left scale), as well as the effective magnetic moment per Co (right scale). These have been evaluated from the SQUID measurements and the XMCD measurements with different applied fields. In addition, the approximation of the effective moment from the contribution of singles, pairs, and triples is shown (dashed line).

these indications, we can conclude that the Co atoms substitute exclusively for $\mathrm{Zn}$ in the lattice. Based on these results of the global and local structural properties, the magnetic properties can solely be discussed with regard to the statistical distribution of Co atoms in the cationic sublattice of the wurtzite crystal structure.

Figure 7 (left scale) displays the abundance of singles, pairs, and triples as given by a statistical dopant distribution in the cationic hcp sublattice using Behringer's equations [35]. Considering the effective spin Hamiltonian for isolated $\mathrm{Co}^{2+}$ dopant atoms [11] together with a Heisenberg-like effective antiferromagnetic coupling of $J / k_{B}=15 \mathrm{~K}$ and a single-ion anisotropy $D / k_{B}=3 \mathrm{~K}$ from Ref. [19] allows one to calculate the expected magnetic moment per Co atom for these small dopant configurations at $2.5 \mathrm{~K}$ and $4 \mathrm{~T}$. Due to the finite external magnetic field and temperature, this yields a magnetic moment for a single Co atom of $3.293 \mu_{B} / \mathrm{Co}$, for a (compensated) pair of $0.059 \mu_{B} / \mathrm{Co}$, for an (unfrustrated) open triple of $1.095 \mu_{B} / \mathrm{Co}$, and for a (frustrated) closed triple of $0.348 \mu_{B} / \mathrm{Co}$, the sum of which results in the dashed line in Fig. 7 (right scale). Obviously, the more atoms we include in the consideration, the lower the effective moment per Co will be. The resulting total effective moment for low Co concentrations is thus dominated by the contribution of singles, whereas with increasing Co percentage the effective moment is strongly reduced due to the vanishing abundance of all small configurations. In addition, it is obvious that the rather low external field assures that the antiferromagnetic pairs as well as the frustrated closed triples remain almost fully in their moment-compensating state since even the field of $4 \mathrm{~T}$ is not sufficient to overcome the weak antiferromagnetic coupling.

Figure 7 (right scale) compiles the experimentally determined effective magnetic moments for a wide concentration range of $\mathrm{Co:ZnO}$ samples. For all samples in Fig. 7, the effective magnetic moment per $\mathrm{Co}$ atom has been quantitatively derived from the measured SQUID magnetization at external magnetic fields of $4 \mathrm{~T}$. The Co concentration has been determined with PIXE and EDX, the thickness was determined by $\mathrm{X}$-ray reflectivity, and the area of the films was directly measured to calculate the volume. These results are given by the open circles. Below the coalescence limit, results of various $\mathrm{Co}: \mathrm{ZnO}$ samples of around $10-15 \%$ of Co doping from Refs. [10,19] are shown. Here singles, pairs, and triples correspond to about half of all existing Co dopant configurations in the sample. Therefore, the deviations between the expected moment for small configurations and the experiment are small. At higher Co concentrations, the effective magnetic moments are systematically above the estimation. To better elaborate the role of the large Co dopant configurations, effective magnetic moments have also been deduced from XMCD experiments at 4.5 T (squares), $6 \mathrm{~T}$ (up triangles), and $17 \mathrm{~T}$ (down triangles). As mentioned above, the size of the XMCD corresponds directly to the effective magnetic moment per Co. If this value is known for one concentration, it is possible to directly correlate the size of the XMCD with an effective moment for all measurements in Fig. 3. Here we have chosen to scale the effective magnetic moment of $3.293 \mu_{B} / \mathrm{Co}$ for $10 \% \mathrm{Co}: \mathrm{ZnO}$, determined by SQUID, to the respective XMCD signal. For all external magnetic fields, the effective moment, determined by XMCD, decreases with increasing Co content. However, the effective magnetic moment at $17 \mathrm{~T}$ is much higher than at low fields. This increase has been used before to extract the strength of the antiferromagnetic coupling constant $J$ [19].

However, for the high Co concentrations studied here, no straightforward modeling of the expected magnetic behavior is possible. As already mentioned, Behringer's equations only allow one to calculate the small dopant configurations, i.e., singles, pairs, and triples [35]. The use of Monte Carlo simulations to derive the abundance for larger dopant configurations has been proposed and tested [45]. However, this approach was also limited to configurations up to 20 dopants and was only tested up to a dopant concentration of $20 \%$. It is obvious that this method also cannot be applied for extreme dopant concentrations well above the coalescence limit, which would require a statistical analysis on huge supercells involving a plethora of partially large dopant configurations. Nevertheless, by preparing Co:ZnO samples over a wide range of concentrations, we are able to experimentally investigate the evolving magnetic properties with increasing Co content, and qualitative conclusions can be drawn. One can consider the difference between the effective moment at 4.5 and $17 \mathrm{~T}$ as a measure of the magnetic behavior of the large Co configurations. At low fields, the singles, pairs, and triples dominate, giving rise to a strong curvature at low magnetic fields, while the larger, partially compensated, and frustrated Co dopant configurations lead to a more or less linear increase with magnetic field. The slope of these decreases with increasing size of the configuration, as discussed for the closed triples and quadruples in Fig. 3 of Ref. [19]. The linear increase in $\mathrm{M}(\mathrm{H})$ at moderate fields $(<20 \mathrm{~T})$ is solely due to the uncompensated fraction of the magnetic moments. Thus, at coalescence, where the configurations are still rather small, the surface of the dopant configurations where the uncompensated moments are located are relatively large compared to the inner volume where the moments are still fully compensated. With increasing Co content, the 
surface-to-volume ratio gets smaller and thus the fraction of compensated moments increases. Since $17 \mathrm{~T}$ are still not sufficient to fully lift this compensation inside the volume of the dopant configuration (which can already be seen easily from the simulation of the pairs), the discrepancy between 4.5 $\mathrm{T}$ and $17 \mathrm{~T}$ decreases for the higher Co concentrations. In other words, while around the coalescence limit a large fraction of the Co moments are still uncompensated and can be aligned by an external magnetic field of $17 \mathrm{~T}$, at high concentrations the fully compensated Co moments inside the volume of a given dopant configuration dominate, which cannot be aligned by the $17 \mathrm{~T}$ to the same extent. Note that the entire discussion of the evolution of the effective magnetic moment is only based on two experimentally verified ingredients: (i) an atomic magnetic moment of $3.293 \mu_{B} /$ Co and a next-cation-neighbor antiferromagnetic coupling $J / k_{B}=15 \mathrm{~K}$. The complexity of the behavior only results in the fact that extreme doping levels are feasible in $\mathrm{Co}: \mathrm{ZnO}$, making this an ideal model system to study coalescence-induced magnetic order in an uncompensated antiferromagnet.

In Fig. 8, the results of all studied magnetic properties are summarized. Figure 8(a) shows the effective magnetic moments deduced from the XMCD measurements at $17 \mathrm{~T}$ for $20 \%$ to $60 \%$ Co: $\mathrm{ZnO}$. At $20 \%$, where still $14 \%$ of the Co atoms are in small configurations of up to 3 Co atoms, the effective magnetic moment is already reduced to around $0.76 \mu_{B} / \mathrm{Co}$ and it reduces further to $0.05 \mu_{B} / \mathrm{Co}$ for $60 \%$, where the small dopant configurations contribute with only $0.002 \%$. Therefore, although the expected decrease of the effective magnetic moment per Co dopant atom cannot be properly calculated, the decrease with increasing Co concentration can be experimentally verified.

As introduced in Ref. [34], uncompensated spins can be pinned while cooling down in external fields if they are

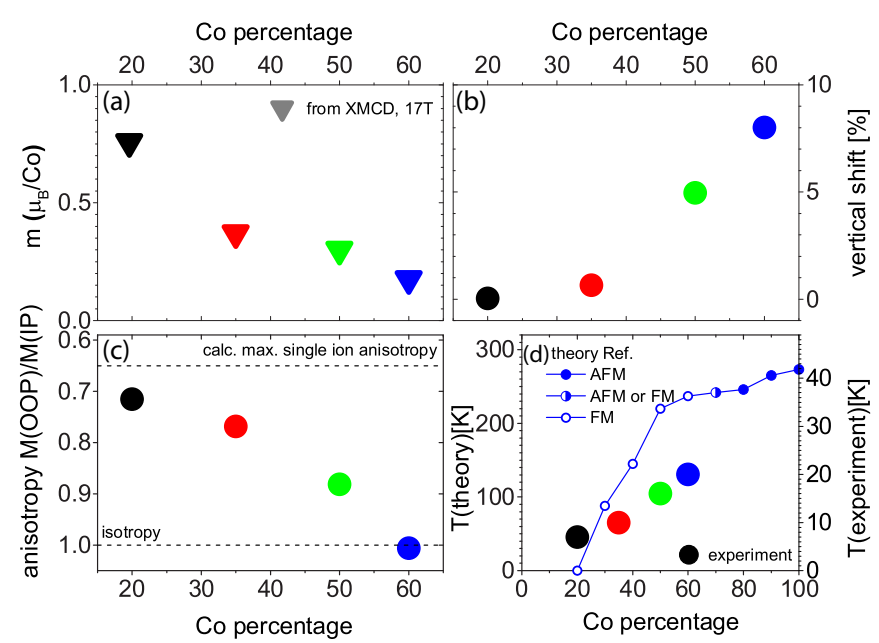

FIG. 8. Summary of results for $\mathrm{Co:ZnO}$. (a) The effective magnetic moment per Co from SQUID at $4 \mathrm{~T}$ and XMCD at $17 \mathrm{~T}$. (b) The vertical exchange-bias shift increases with Co percentage, whereas (c) the anisotropy of the samples decreases with increasing Co percentage. The calculated maximum single-ion anisotropy and isotropy are marked. The comparison in (d) shows the theoretical results of Ref. [26] and our experimental results for the order temperature of $\mathrm{Co}: \mathrm{ZnO}$. magnetically coupled to sufficiently large volumes of magnetically compensated dopant configurations. This pinning results in a vertical exchange bias, the size of which is summarized in Fig. 8(b). A large number of sufficiently large antiferromagnetic configurations, as present for high Co concentrations, results in a pinning of a large number of uncompensated moments and thus a large vertical shift of the hysteresis. In turn, small compensated dopant configurations as dominant for lower Co concentrations cannot pin their respective uncompensated fraction of the moment and thus no $(20 \%)$ or little $(35 \%)$ vertical exchange bias is observed.

Turning to the magnetic anisotropy, in Fig. 8(c), the anisotropy $\mathrm{M}(\mathrm{OOP}) / \mathrm{M}(\mathrm{IP})$ as determined by SQUID is compared to the calculated maximum single-ion anisotropy of 0.65 , which was derived using $D / k_{B}=3 \mathrm{~K}, T=2.5 \mathrm{~K}$, $J / k_{B}=15 \mathrm{~K}, S=3 / 2 ; g_{\perp}=2.276$. For $20 \% \mathrm{Co}: \mathrm{ZnO}$, the experimental value is 0.71 , which is slightly less anisotropic than for $10 \%$ and $15 \%$ of Co doping [10], respectively. With increasing Co percentage, the films become more isotropic and gradually approach the isotropic value of 1 for $60 \% \mathrm{Co}: \mathrm{ZnO}$. It has already been observed that the single-ion anisotropy $D$ apparently decreases from $4 \mathrm{~K}$ at a few $\%$ of Co doping [7] to 3 and $2 \mathrm{~K}$ for $10 \%$ and $15 \%$ of Co doping, respectively [10]. Given the fact that the loss in anisotropy is mainly due to a loss in the curvature of the $\mathrm{M}(\mathrm{H})$ for IP and that frustrated Co dopant configurations behave virtually isotropic at low magnetic fields [19], the previously observed apparent reduction of $D$ in Ref. [10] may have already been caused by the increasing contribution of larger dopant configurations which were neglected for the low Co concentrations in the previous studies. Obviously, the overall magnetic behavior at high Co concentrations is no longer governed by the on-site single-ion anisotropy, but by the next-cation-neighbor antiferromagnetic interaction with its resulting (partial) magnetic frustration. This has consistently been reflected by the decrease in effective moment per Co atom, the loss in anisotropy, and the increase in the vertical exchange-bias shift summarized in Figs. 8(a)-8(c).

Finally, in Fig. 8(d) (right scale), the experimental results for the characteristic ordering temperature shown in Fig. 6 are compared to theoretical results from Ref. [26] (left scale). Theory has predicted ferromagnetic ordering from just above coalescence, i.e., $20 \%$ to $60 \%$, with a strongly increasing order temperature. In contrast, the experimental results show a much less pronounced increase of the order temperature from no order at $20 \%$ to just above $20 \mathrm{~K}$ for the $60 \%$ sample. In addition, no ferromagnetic contribution was observable with SQUID or with XMCD. All films remain antiferromagnetic with an increasing degree of magnetic compensation. The experimental fact that even $60 \% \mathrm{Co}: \mathrm{ZnO}$ only orders antiferromagnetically at low temperatures more closely resembles the behavior of the antiferromagnetic spinel $\mathrm{Co}_{3} \mathrm{O}_{4}$, which has a Néel temperature of $40 \mathrm{~K}$ [46], than the behavior of cubic $\mathrm{CoO}$, the Néel temperature of which is close to room temperature and thus similar to the calculated one of wurtzite $\mathrm{CoO}$ in Ref. [26]. It should be noted that the calculations of Ref. [26] have taken into account effective exchange integrals up to rather distant $(\sim 7.67 \AA)$ neighbors, however, within the CPA. Some of the exchange integrals 
change their sign, i.e., they alter between ferromagnetic and antiferromagnetic as a function of Co concentration, which gives rise to the ferromagnetic region in the theoretical phase diagram. The absence of a ferromagnetic phase in the experimental results presented here may indicate that the theory could overestimate the strength of the distant-neighbor interactions. Alternatively, the disorder of the dopant together with the resulting magnetic frustration, which has been evidenced by the training effect upon FC in Ref. [34], plays a more decisive role for the magnetic properties, which is to a certain extent neglected in the CPA of the theory. In any case, the magnetic properties for $\mathrm{Co}: \mathrm{ZnO}$ from $20 \%$ to $60 \%$ seem to be dominated by a short-range, antiferromagnetic, next-cationneighbor exchange interaction and the order temperature does not significantly exceed the previously reported value of $15 \mathrm{~K}$ for lower Co concentration. This can be taken as experimental evidence that distant-neighbor interactions are not governing the magnetic properties of $\mathrm{Co}: \mathrm{ZnO}$ over a wide range of doping concentrations. It may be interesting for future studies whether the alternation of the carrier concentration may alter these findings since all samples studied here were rather resistive.

\section{CONCLUSION}

The evolution of structural and magnetic properties of Co doped $\mathrm{ZnO}$ has been studied over a wide concentration range from $20 \%$ up to $60 \%$ while retaining the wurtzite crystal structure of the $\mathrm{ZnO}$ host crystal. The formation of secondary phases, in particular metallic Co precipitations, can be excluded and Co exclusively substitutes for $\mathrm{Zn}$ and remains in its formal $2+$ oxidation state. A short-range, antiferromagnetic, next-cation-neighbor exchange interactions leads to an increasing degree of magnetic compensation, while uncompensated spins cause the occurrence of a hysteresis at low temperatures. As a result, $\mathrm{Co}: \mathrm{ZnO}$ is an uncompensated antiferromagnet with an order temperature which increases only to $20 \mathrm{~K}$ with increasing Co concentration. This is accompanied by a continuous loss of single-ion anisotropy, a reduced effective magnetic moment per $\mathrm{Co}$, and an increasing vertical exchange-bias shift.

\section{ACKNOWLEDGMENT}

We want to thank the Austrian Science Fund (FWF), Grant No. P26164-N20, for funding.
[1] T. Dietl, H. Ohno, F. Matsukura, J. Cibert, and D. Ferrand, Science 287, 1019 (2000).

[2] K. Ueda, H. Tabata, and T. Kawai, Appl. Phys. Lett. 79, 988 (2001).

[3] V. Ney, S. Ye, T. Kammermeier, A. Ney, H. Zhou, J. Fallert, H. Kalt, F.-Y. Lo, A. Melnikov, and A. D. Wieck, J. Appl. Phys. 104, 083904 (2008).

[4] K. Potzger, Shengqiang Zhou, F. Eichhorn, M. Helm, W. Skorupa, A. Mücklich, J. Fassbender, T. Herrmannsdörfer, and A. Bianchi, J. Appl. Phys. 99, 063906 (2006).

[5] P. Sharma, A. Gupta, K. V. Rao, F. J. Owens, R. Sharma, R. Ahuja, J. M. O. Guillen, B. Johansson, and G. A. Gehring, Nat. Mater. 2, 673 (2003).

[6] K. R. Kittilstved, D. A. Schwartz, A. C. Tuan, S. M. Heald, S. A. Chambers, and D. R. Gamelin, Phys. Rev. Lett. 97, 037203 (2006).

[7] P. Sati, R. Hayn, R. Kuzian, S. Régnier, S. Schäfer, A. Stepanov, C. Morhain, C. Deparis, M. Laügt, M. Goiran, and Z. Golacki, Phys. Rev. Lett. 96, 017203 (2006).

[8] A. Barla, G. Schmerber, E. Beaurepaire, A. Dinia, H. Bieber, S. Colis, F. Scheurer, J.-P. Kappler, P. Imperia, F. Nolting, F. Wilhelm, A. Rogalev, D. Müller, and J. J. Grob, Phys. Rev. B 76, 125201 (2007).

[9] A. Ney, K. Ollefs, S. Ye, T. Kammermeier, V. Ney, T. C. Kaspar, S. A. Chambers, F. Wilhelm, and A. Rogalev, Phys. Rev. Lett. 100, 157201 (2008).

[10] A. Ney, T. Kammermeier, K. Ollefs, S. Ye, V. Ney, T. C. Kaspar, S. A. Chambers, F. Wilhelm, and A. Rogalev, Phys. Rev. B 81, 054420 (2010).

[11] T. L. Estle and M. de Wit, Bull. Am. Phys. Soc. 6, 445 (1961).

[12] P. Koidl, Phys. Rev. B 15, 2493 (1977).

[13] R. O. Kuzian, A. M. Daré, P. Sati, and R. Hayn, Phys. Rev. B 74, 155201 (2006).
[14] S. W. Yoon, S.-B. Cho, S. C. We, S. Yoon, B. W. Suh, H. K. Song, and J. J. Shin, J. Appl. Phys. 93, 7879 (2003).

[15] S. Kolesnik, B. Dabrowski, and J. Mais, J. Appl. Phys. 95, 2582 (2004).

[16] M. Bouloudenine, N. Viart, S. Colis, J. Kortus, and A. Dinia, Appl. Phys. Lett. 87, 052501 (2005).

[17] G. Lawes, A. S. Risbud, A. P. Ramirez, and R. Seshadri, Phys. Rev. B 71, 045201 (2005).

[18] P. Sati, C. Deparis, C. Morhain, S. Schäfer, and A. Stepanov, Phys. Rev. Lett. 98, 137204 (2007).

[19] A. Ney, V. Ney, F. Wilhelm, A. Rogalev, and K. Usadel, Phys. Rev. B 85, 245202 (2012).

[20] T. Chanier, M. Sargolzaei, I. Opahle, R. Hayn, and K. Koepernik, Phys. Rev. B 73, 134418 (2006).

[21] T. C. Kaspar, T. Droubay, S. M. Heald, M. H. Engelhard, P. Nachimuthu, and S. A. Chambers, Phys. Rev. B 77, 201303 (2008).

[22] A. Ney, M. Opel, T. C. Kaspar, V. Ney, S. Ye, K. Ollefs, T. Kammermeier, S. Bauer, K.-W. Nielsen, S. T. B. Goennenwein, M. H. Engelhard, S. Zhou, K. Potzger, J. Simon, W. Mader, S. M. Heald, J. C. Cezar, F. Wilhelm, A. Rogalev, R. Gross, and S. A. Chambers, New J. Phys. 12, 013020 (2010).

[23] A. Ney, A. Kovács, V. Ney, S. Ye, K. Ollefs, T. Kammermeier, F. Wilhelm, A. Rogalev, and R. E. Dunin-Borkowski, New J. Phys. 13, 103001 (2011).

[24] B. B. Straumal, A. A. Mazilkin, S. G. Protasova, P. B. Straumal, A. A. Myatiev, G. Schütz, E. Goering, T. Tieze, and B. Baretzky, Philos. Mag. 93, 1371 (2013).

[25] V. Ney, K. Lenz, K. Ollefs, F. Wilhelm, A. Rogalev, and A. Ney, J. Appl. Phys. 116, 043912 (2014).

[26] S. K. Nayak, M. Ogura, A. Hucht, H. Akai, and P. Entel, J. Phys.: Condens. Matter 21, 064238 (2009). 
[27] C. D. Lorenz, R. May, and R. M. Ziff, J. Stat. Phys. 98, 961 (2000).

[28] M. J. Redman and E. G. Steward, Nature (London) 193, 867 (1962).

[29] J. Alaria, N. Cheval, K. Rode, M. Venkatesan, and J. M. D. Coey, J. Phys. D: Appl. Phys. 41, 135004 (2008).

[30] T. Dietl, T. Andrearczyk, A. Lipinska, M. Kiecana, Maureen Tay, and Yihong Wu, Phys. Rev. B 76, 155312 (2007).

[31] A. Ney, V. Ney, M. Kieschnick, F. Wilhelm, K. Ollefs, and A. Rogalev, J. Appl. Phys. 115, 172603 (2014).

[32] M. Sawicki, E. Guziewicz, M. I. Lukasiewicz, O. Proselkov, I. A. Kowalik, W. Lisowski, P. Dluzewski, A. Wittlin, M. Jaworski, A. Wolska, W. Paszkowicz, R. Jakiela, B. S. Witkowski, L. Wachnicki, M. T. Klepka, F. J. Luque, D. Arvanitis, J. W. Sobczak, M. Krawczyk, A. Jablonski, W. Stefanowicz, D. Sztenkiel, M. Godlewski, and T. Dietl, Phys. Rev. B 88, 085204 (2013).

[33] B. Henne, V. Ney, K. Ollefs, F. Wilhelm, A. Rogalev, and A. Ney, Sci. Rep. 5, 16863 (2015).

[34] B. Henne, V. Ney, M. de Souza, and A. Ney, Phys. Rev. B 93, 144406 (2016).

[35] R. E. Behringer, J. Chem. Phys. 29, 537 (1958).
[36] A. Kovács, A. Ney, M. Duchamp, V. Ney, C. B. Boothroyd, P. L. Galindo, T. C. Kaspar, S. A. Chambers, and R. E. Dunin-Borkowski, J. Appl. Phys. 114, 243503 (2013).

[37] A. Ney, T. Kammermeier, V. Ney, K. Ollefs, and S. Ye, J. Magn. Magn. Mater. 320, 3341 (2008).

[38] A. Ney, Semicond. Sci. Technol. 26, 064010 (2011).

[39] M. Sawicki, W. Stefanowicz, and A. Ney, Semicond. Sci. Technol. 26, 064006 (2011).

[40] A. Rogalev, J. Goulon, C. Goulon-Ginet, and C. Malgrange, Lect. Notes Phys. 565, 60 (2001).

[41] A. Rogalev, F. Wilhelm, J. Goulon, and G. Goujon, in Magnetism and Synchrotron Radiation: Towards the Fourth Generation Light Sources, Springer Proceedings in Physics, Vol. 151 (Springer, Switzerland, 2013), pp. 289-314.

[42] JCPDS Card No. 89-1397 (unpublished).

[43] E. H. Kisi and M. M. Elcombe, Acta Cryst. C 45, 1867 (1989).

[44] A. Ney, V. Ney, K. Ollefs, D. Schauries, F. Wilhelm, and A. Rogalev, J. Surf. Interfac. Mater. 2, 14 (2014).

[45] T. C. Droubay, T. C. Kaspar, B. P. Kaspar, and S. A. Chambers, Phys. Rev. B 79, 075324 (2009).

[46] W. L. Roth, J. Phys. Chem. Solids 25, 1 (1964). 